\title{
Chiral-Like Critical Behavior in the Antiferromagnet Cobalt Glycerolate
}

\author{
F. L. Pratt, ${ }^{1}$ P. J. Baker, ${ }^{2}$ S. J. Blundell, ${ }^{2}$ T. Lancaster, ${ }^{2}$ M. A. Green, ${ }^{3}$ and M. Kurmoo ${ }^{4}$ \\ ${ }^{1}$ ISIS Facility, Rutherford Appleton Laboratory, Chilton, Oxfordshire OX11 OQX, United Kingdom \\ ${ }^{2}$ University of Oxford, Department of Physics, Clarendon Laboratory, Parks Road, Oxford OX1 3PU, United Kingdom \\ ${ }^{3}$ NIST Center for Neutron Research, 100 Bureau Drive, Gaithersburg, Maryland 20899-2330, USA \\ ${ }^{4}$ Laboratoire de Chimie de Coordination Organique, CNRS-UMR7140, Université Louis Pasteur, 67000 Strasbourg, France
}

(Received 9 January 2007; published 3 July 2007)

\begin{abstract}
Critical exponents closely matching those of the $N=2$ chiral universality class have been obtained for the layered magnetic system cobalt glycerolate using muon spin relaxation. This class was originally introduced to represent geometrically frustrated triangular stacked-layer $X Y$ magnets with chiral noncollinear spin structures. Since the present magnetic system is a canted $X Y$ system without geometrical frustration or chiral degeneracy, the results indicate that the order parameter for canting in this system plays a similar role to the chiral order parameter in the geometrically frustrated systems, strongly suggesting that both types of noncollinear system share the same universality class.
\end{abstract}

DOI: 10.1103/PhysRevLett.99.017202

PACS numbers: 75.40.Cx, 75.25.+z, 75.40.Gb, 76.75.+i

The study of the critical behavior of continuous magnetic transitions near the ordering temperature using techniques such as neutron scattering has proved to be a powerful way of determining the nature and symmetry of magnetic interactions [1]. Based on the concepts of critical scaling and universality, theoretical results for the asymptotic power-law critical exponents have now been calculated for model systems from a variety of universality classes [2] and these can be compared directly with experiments. One of the more recent classes to emerge is the socalled $N=2$ chiral (N2C) universality class [3], first identified by Kawamura [4] in the context of stacked triangular antiferromagnets (STAs) with easy-plane anisotropy, whose properties are dominated by frustration and noncollinear magnetic order. The proposed order parameter symmetry $Z_{2} \times S_{1}$ reflects twofold chiral $\left(Z_{2}\right)$ and continuous $X Y\left(S_{1}\right)$ components. Corresponding critical exponents have been calculated in a number of studies [5-9], converging on values significantly different from those of standard collinear magnets and in rather good agreement with experiments on the easy-plane STA CsMnBr $3[3,10]$. So far this STA is the best example to show N2C behavior and finding further clear examples of this $\mathrm{N} 2 \mathrm{C}$ class is desirable to fully establish the validity and scope of the class. This has been questioned recently following theoretical [11] and experimental [12] studies indicating that transitions in some N2C candidate systems of the STA type are weakly first order. In this Letter, we report on cobalt glycerolate, $\mathrm{Co}\left(\mathrm{C}_{3} \mathrm{H}_{6} \mathrm{O}_{3}\right)$ (CoGly) [13,14], an example of a layered antiferromagnet (AF) with a noncollinear spin structure. Lacking geometric frustration and chiral degeneracy, it nevertheless is found to show N2C criticality.

The sample used for the measurements was prepared at the University of Strasbourg and the $\mu$ SR measurements were carried out at two complementary $\mu$ SR facilities. Zero field (ZF) relaxation and transverse field (TF) rotation studies were carried out at the ISIS Muon Facility in the
UK using, respectively, the MUSR and ARGUS instruments. For ZF precession studies of the magnetically ordered state requiring fast time resolution, the GPS instrument at the $S \mu S$ facility of the Paul Scherrer Institut, Switzerland was used.

The crystal structure of CoGly is monoclinic [13], consisting of puckered cobalt oxide magnetic layers parallel to the $b c$ plane, sandwiched by the $\mathrm{CH}_{2} \mathrm{CHCH}_{2}$ organic backbone of the glycerol, with the separation between magnetic layers along the $a$ axis being $0.8 \mathrm{~nm}$. The magnetic susceptibility above $100 \mathrm{~K}$ follows the Curie-Weiss law with an AF Weiss constant $\theta=-55 \mathrm{~K}$ [15] and magnetic ordering takes place below $T_{N} \sim 35.5 \mathrm{~K}$. The structure within a layer (Fig. 1) consists of a distorted hexagonal network of magnetic $\mathrm{Co}^{2+}$ ions connected by single $\left(J_{1}\right)$ and double $\left(J_{2}\right)$ oxygen bridges. The $J_{1}$ bridges have a Co$\mathrm{O}-\mathrm{Co}$ angle of $133^{\circ}$, suggesting AF exchange, whereas the $J_{2}$ bridges have an angle close to $90^{\circ}\left(98^{\circ}\right)$, suggesting weaker ferromagnetic (FM) exchange.

The structure has been measured at $1.9 \mathrm{~K}$ using the D20 neutron diffractometer at the ILL in Grenoble, France and the diffraction data are shown in Fig. 2. A wavelength of $2.418 \AA$ was used and the data were analyzed using the GSAS suite of programs [16]. The space group is $P 2_{1} / c$ with lattice parameters $a=8.106(9), b=6.398(10), c=$ 8.643(7) $\AA$, and $\beta=93.60(9)^{\circ}$. The magnetic cell is $\left(\begin{array}{lll}2 a & b & c\end{array}\right)$ with a moment of 2.27(5) $\mu_{B}$ on each cobalt. The goodness-of-fit parameters were $R_{\mathrm{wp}}=0.54 \%$ and $R_{p}=0.37 \%$. Spin orientations were determined to a precision of $3^{\circ}$ and the resulting spin structure is shown in Fig. 1. As expected, AF coupled chains run along $b$ with FM coupling in the $c$ direction; however, each chain is canted out of plane by $19^{\circ}$. The canting direction alternates for successive chains so that the overall structure within a layer is still AF. The only simple collinear interactions in this system are those in the weakly coupled interlayer direction $a$. The orientation of the AF chains within the 

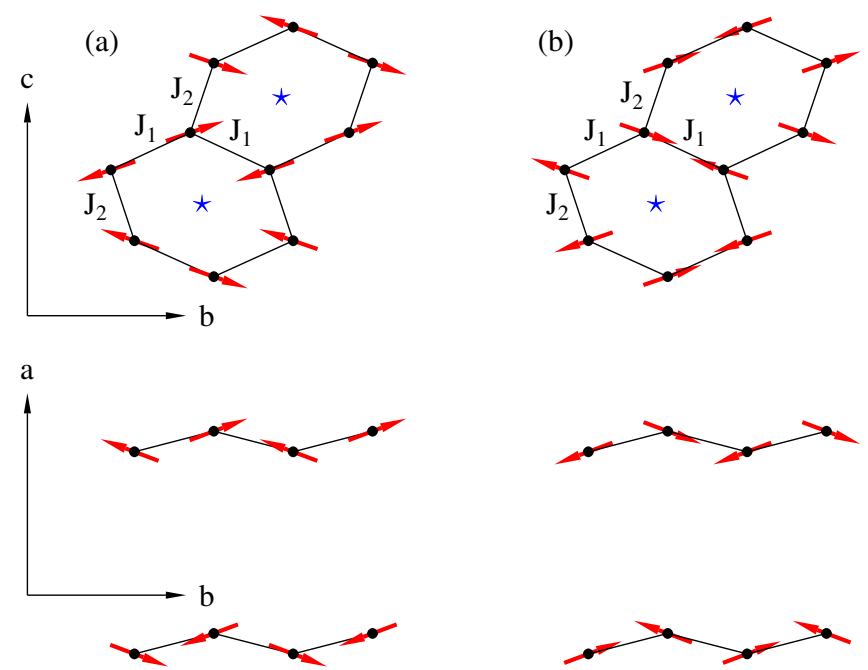

FIG. 1 (color online). The ordered spin structure of CoGly derived from neutron diffraction. Co spins within one layer form a distorted hexagonal network when projected onto the $b c$ plane. The spins can be viewed as forming chains along the $b$ axis following dominant $\mathrm{AF}$ coupling $J_{1}$; however, each $\mathrm{AF}$ chain is canted by $19^{\circ}$ out of the plane and its easy axis within the plane follows closely one of the $J_{1}$ directions. The coupling between adjacent chains resulting from $J_{2}$ is dominantly FM. (a) and (b) show two equivalent spin structures reflecting local alignment with one or other of the $J_{1}$ interaction paths. Stars indicate the predominant muon sites assigned to $f_{1}$.

$b c$ plane appears to follow closely one of the two $J_{1}$ couplings present at each Co site and there are thus two possible noncollinear structures resulting from this degeneracy [Fig. 1(a) and 1(b)] produced by competition between the local anisotropy at the Co sites and the exchange couplings $J_{1}$ and $J_{2}$.

For critical studies of AF systems, a local probe technique such as muon spin relaxation ( $\mu \mathrm{SR})$ [17] can be very useful as it is usually directly sensitive to the staggered order parameter, in marked contrast to bulk magnetic measurements. $\mu \mathrm{SR}$ also has the advantage that it does

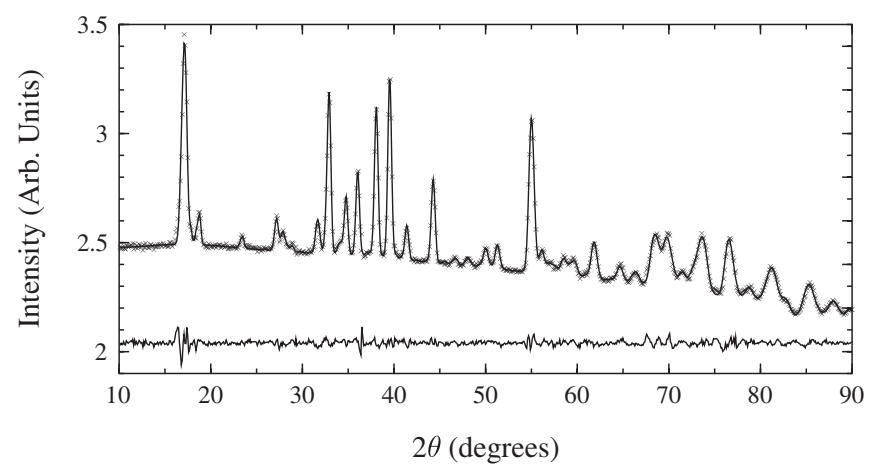

FIG. 2. The neutron powder diffraction pattern of CoGly measured at $1.9 \mathrm{~K}$ (symbols) along with the simulation obtained from the data analysis (line). The lower curve shows the difference between the data and the simulation. not generally need to use large single crystals. A number of previous $\mu$ SR studies have looked at magnetic critical exponents. Although some earlier reports concerned AF systems [18], recent attention has mainly been focused on FM examples [19-21]. In the AF case, a reasonably broad and accessible critical region allows systematic analysis of critical behavior using $\mu$ SR and we demonstrate here a method for extracting the full set of critical exponents. First, exponent $\beta$ is obtained from the zero field $\mu \mathrm{SR}$ precession in the magnetically ordered state. The $\mu \mathrm{SR}$ frequency shift in a small transverse field gives the second exponent $\gamma$. Finally, the longitudinal muon spin relaxation provides the exponent $w$. Since the exponents are related by a set of scaling relations [2], these three exponents measured by $\mu$ SR should be sufficient to determine the complete set of static and dynamic critical exponents.

Example ZF muon data are shown in Fig. 3(a), just above and just below $T_{N}$. A well-defined $\mathrm{ZF}$ precession signal is clearly seen in the ordered state, whose dominant frequency $f_{1}$ reaches $54 \mathrm{MHz}$ at the lowest $T$. The Fourier spectrum of the precession well below $T_{N}$ also shows three weaker components at lower frequency [Fig. 3(b) and 3(c)]. The $T$ dependence of $f_{1}$ reflects the magnetic order parameter [Fig. 4(a)]. The critical exponent $\beta$ is extracted from the data near the transition [Fig. 4(a) inset], which follows $f_{1} \propto\left[1-\left(T / T_{N}\right)^{n}\right]^{\beta}$, where $n$ is close to 1 , giving $\beta=0.228(9)$. This is significantly lower than the value 0.346 for a standard 3D $X Y$ system and reasonably close to the averaged estimate of $0.25(1)$ for Monte Carlo (MC) simulations of the N2C class (Table I).

The presence of a dominant single frequency within a complex magnetic structure strongly suggests a primary muon site with high symmetry. Following dipolar field calculation for the spin structure of Fig. 1, the site $(0.5,0,0)$ is assigned to $f_{1}$. Taking the Co moment seen by neutrons, this site has muon precession frequency $54.3 \mathrm{MHz}$, closely matching the measured $f_{1}$. The site sits at the center of the distorted hexagons within the
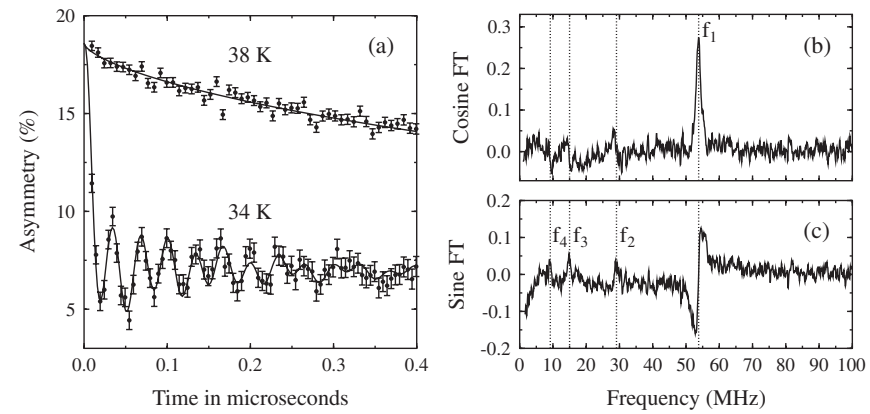

FIG. 3. (a) ZF muon spin relaxation data for CoGly both above and below $T_{N}$, with lines as a guide to the eye. (b) and (c) show the cosine and sine Fourier transforms of the precession data obtained below $16 \mathrm{~K}$. Four main frequencies can be resolved, with the dominant one at $54 \mathrm{MHz}\left(f_{1}\right)$ and three others at lower frequency $\left(f_{2}\right.$ to $\left.f_{4}\right)$. 

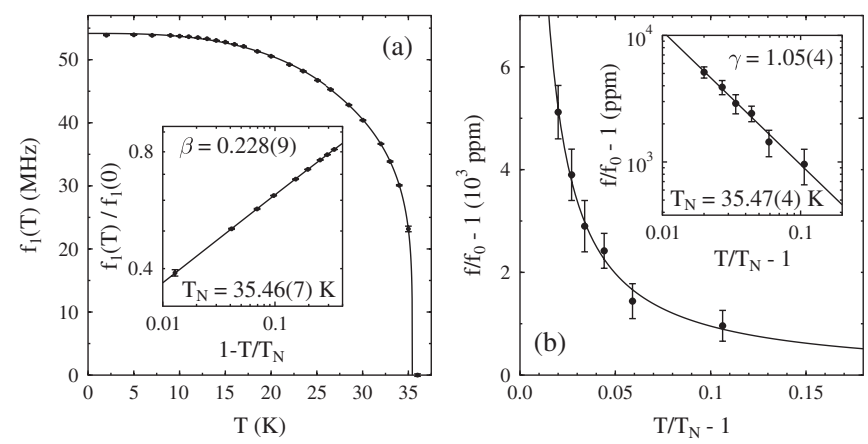

FIG. 4. (a) The $T$ dependence of the dominant ZF precession frequency $f_{1}$ (the line is a guide to the eye). The inset shows the scaling plot from which $\beta$ is determined. (b) Frequency shift of the muon spin rotation in a TF of $5 \mathrm{mT}$. The inset shows the scaling plot used for deriving $\gamma . T_{N}$ was determined from the transition midpoint.

magnetic layer (shown as stars in Fig. 1). Sites with lower frequencies matching $f_{2}$ to $f_{4}$ can be found in the region between the magnetic layers.

By applying a small TF in the paramagnetic phase, a measurement of the $T$ dependence of the local magnetic susceptibility may be obtained from the shift of the muon spin rotation frequency $f / f_{0}-1 \propto \chi \propto\left|T / T_{N}-1\right|^{-\gamma}$. From this the critical exponent $\gamma$ is obtained [Fig. 4(b)]. The resulting value $\gamma=1.05(4)$ is significantly smaller than 1.318, the standard value expected for a $3 \mathrm{D} X Y$ system, but consistent with the average MC prediction 1.07(3) for the N2C class (Table I).

Further static exponents $\alpha, \nu$, and $\eta$ can be derived from the measured $\beta$ and $\gamma$ using the scaling relation

$$
\alpha=2-2 \beta-\gamma,
$$

and hyperscaling relations involving the spatial dimension $d=3$ :

$$
\nu=(2-\alpha) / d=(2 \beta+\gamma) / d
$$

TABLE I. Static critical exponents obtained from analysis of the $\mu$ SR data for CoGly compared with theoretical predictions for different 3D magnetic models. The values of $\beta$ and $\gamma$ for CoGly have been obtained directly from the measurements and the values of $\nu$ and $\alpha$ and $\eta$ have been derived from scaling relations. Experimental values from neutron scattering on the $X Y$ STA CsMnBr 3 are also provided for comparison.

\begin{tabular}{llllll}
\hline \hline & \multicolumn{1}{c}{$\beta$} & \multicolumn{1}{c}{$\gamma$} & \multicolumn{1}{c}{$\nu$} & \multicolumn{1}{c}{$\alpha$} & \multicolumn{1}{c}{$\eta$} \\
\hline CoGly & $0.228(9)$ & $1.05(4)$ & $0.50(1)$ & $0.49(4)$ & $-0.09(5)$ \\
N2C (MC) [22] & $0.25(1)$ & $1.07(3)$ & $0.51(1)$ & $0.37(5)$ & $-0.07(10)$ \\
N2C (FT) [8] & $0.31(5)$ & $1.10(4)$ & $0.57(3)$ & $0.29(9)$ & $0.09(1)$ \\
$X Y$ [2] & 0.346 & 1.318 & 0.672 & -0.015 & 0.038 \\
Heisenberg [2] & 0.369 & 1.396 & 0.711 & -0.134 & 0.038 \\
O(4) [23] & 0.39 & 1.39 & 0.70 & -0.17 & 0.02 \\
CsMnBr [24] $_{3}$ & $0.21(2)$ & $1.01(8)$ & $0.54(3)$ & $0.57(9)$ & $0.1(2)$ \\
CsMnBr $_{3}$ [25] & $0.25(2)$ & $1.10(5)$ & $0.57(3)$ & $0.40(5)$ & $0.07(13)$ \\
\hline \hline
\end{tabular}

$$
\eta=2-\gamma / \nu=2-\gamma d /(2 \beta+\gamma) .
$$

The values obtained for all these critical exponents are well matched to $\mathrm{N} 2 \mathrm{C}$ class predictions (Table I), whereas the standard $X Y(N=2)$, Heisenberg $(N=3)$ and $\mathrm{O}(4)(N=$ 4) classes are clearly inconsistent with the measurements. The experimental values are also similar to those obtained from neutron scattering on the $X Y$ STA $\mathrm{CsMnBr}_{3}$. Both $\mu \mathrm{SR}$ and MC values of $\eta$ derived using (3) are slightly negative, whereas $\eta$ should always be positive for a continuous transition [2]. This probably reflects the limited $T$ ranges and model sizes involved in the scaling analysis, leading to corrections to scaling that produce slightly modified effective exponents. Whereas the scaling relation (1) remains robust against these corrections, the hyperscaling relations (2) and (3) acquire additional errors [26], that will be particularly significant for $\eta$, as it is so close to zero. It is notable that direct calculation of $\eta$ using field theory (FT) has given the positive value 0.09(1) [8] (Table I) and this is expected to be more reliable than values dependent on hyperscaling.

The longitudinal muon spin relaxation is sensitive to the dynamic critical behavior, probing the spin correlation time $\tau$, which diverges as $\tau \propto\left|T-T_{N}\right|^{-w}$ following the slowing down of critical fluctuations near the transition. In the fast fluctuation regime that applies here the relaxation rate $\lambda$ is directly proportional to $\tau$ and thus also follows the critical exponent $w$. This is related to the dynamic exponent $z$ and two static exponents by [27]

$$
w=\nu(z+2-d-\eta) .
$$

Divergence of the measured $\mathrm{ZF}$ relaxation is clearly seen, both above and below the transition [Fig. 5(a)]. A secondary relaxation peak also appears near $10 \mathrm{~K}$ [Fig. 5(a)]; however, no anomaly is seen in $f_{1}$ near $10 \mathrm{~K}$ [Fig. 4(a)], so there can be no significant change in the spin structure. A similar feature is seen in the ac susceptibility [15], most likely related to the motion of AF domains. From the scaling analysis of $\lambda$ near $T_{N}$ [Fig. 5(b)] it is found that one value $w=0.20(1)$ describes the data both above and below $T_{N}$. No cutoff to the scaling is seen near the transition, in contrast to the dipolar crossover typically seen for FM systems [21]. The ratio of $\lambda$ values above and below the transition for the same value of $\left|T-T_{N}\right|$ is 5.4(2), close to the universal susceptibility ratio, which is calculated to be in the range 4.7-5.0 [2]. To derive $z$ from the measured $\mu \mathrm{SR}$ exponents, we combine (2)-(4), to give

$$
z=d(2 \beta+w) /(2 \beta+\gamma) \text {. }
$$

This results in the value $z=1.25(6)$, which is smaller than the standard value $z=d / 2=1.5$, expected for an $\mathrm{AF}$ [27]. The breakdown of hyperscaling giving the apparently negative value for $\eta$ is probably also the main origin of this discrepancy. If, on the other hand, we assume the standard value $z=1.5$, an alternative estimate of $\eta$ can be provided by (4). The positive value $\eta=0.10$ (3) is then obtained, 

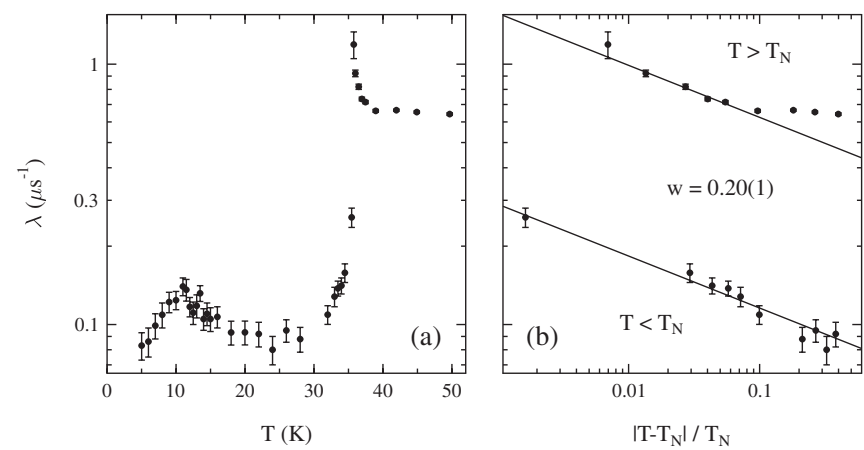

FIG. 5. (a) The $T$ dependence of the ZF longitudinal relaxation rate $\lambda$. (b) Scaling plot showing the critical behavior of $\lambda$ on approaching the transition at $T_{N}=35.52(3) \mathrm{K}$ from the paramagnetic region (upper data) and the ordered region (lower data). The fitted $\lambda$ on the paramagnetic side is a factor 5.4(2) greater than that of the ordered side.

fully consistent with the $\mathrm{N} 2 \mathrm{C}$ value $0.09(1)$ obtained from field theory.

From Table I, both CoGly and $\mathrm{CsMnBr}_{3}$ are seen to be very good experimental examples of $\mathrm{N} 2 \mathrm{C}$ criticality. It appears that in easy-plane layered systems, both the chiral and canting degrees of freedom are working in a similar way. This is not unreasonable, since both are manifestations of noncollinearity that can be represented by a twofold $Z_{2}$ component in the order parameter, thus transitions of both types would be expected to share the same universality class. Further evidence for N2C behavior in the absence of chirality has also been highlighted in MC simulation [9]. One is drawn to the overall conclusion that, as far as the spin correlations are concerned, it is the noncollinearity resulting from the competing spin interactions that is the defining feature of these $\mathrm{N} 2 \mathrm{C}$ systems, rather than the specific property of chirality. Therefore, the class would perhaps better be named the $N=2$ noncollinear (N2NC) class.

In conclusion, $\mu$ SR has been shown to be a powerful probe of the critical magnetic properties in this particular $\mathrm{AF}$, demonstrating N2NC-class critical behavior in a system that is very different from the STA and helimagnet systems that have previously been studied as representatives of this class. The result therefore provides further support to the idea of universal behavior among such systems and suggests that there should perhaps be wider relevance within magnetism for this $\mathrm{N} 2 \mathrm{NC}$ universality class than previously acknowledged.

The authors thank Alex Amato for instrument support at PSI and the EPSRC and CNRS for financial support. T. L. thanks the Royal Commission for the Exhibition of 1851 for financial support. This research project has been supported by the EC under the 6th Framework Programme through the Key Action: Strengthening the European
Research Area, Research Infrastructures, Contract No. RII3-CT-2003-505925.

[1] M.F. Collins, Magnetic Critical Scattering (Oxford University, New York, 1989).

[2] A. Pelissetto and E. Vicari, Phys. Rep. 368, 549 (2002).

[3] H. Kawamura, J. Phys. Condens. Matter 10, 4707 (1998).

[4] H. Kawamura, J. Phys. Soc. Jpn. 58, 584 (1989).

[5] H. Kawamura, J. Phys. Soc. Jpn. 61, 1299 (1992).

[6] M. L. Plumer and A. Mailhot, Phys. Rev. B 50, 16113 (1994).

[7] E. H. Boubcheur, D. Loison, and H. T. Diep, Phys. Rev. B 54, 4165 (1996).

[8] A. Pelissetto, P. Rossi, and E. Vicari, Phys. Rev. B 63, 140414(R) (2001).

[9] M. Žukovič, T. Idogaki, and K. Takeda, Phys. Rev. B 63, 172412 (2001).

[10] M.F. Collins and O. A. Petrenko, Can. J. Phys. 75, 605 (1997).

[11] B. Delamotte, D. Mouhanna, and M. Tissier, Phys. Rev. B 69, 134413 (2004).

[12] G. Quirion, X. Han, M. L. Plumer, and M. Poirier, Phys. Rev. Lett. 97, 077202 (2006).

[13] P. G. Slade, E. W. Radoslovich, and M. Raupach, Acta Crystallogr., B 27, 2432 (1971).

[14] R. P. Eckberg, W.E. Hatfield, and B. D. Losee, Inorg. Chem. 13, 740 (1974).

[15] M. Kurmoo et al. (unpublished).

[16] A. C. Larson and R. B. Von Dreele, "General Structure Analysis System (GSAS)," Los Alamos National Laboratory, Report No. LAUR 86-748, 2003.

[17] S. J. Blundell, Contemp. Phys. 40, 175 (1999).

[18] R. De Renzi, G. Guidi, P. Podini, R. Tedeschi, C. Bucci, and S. F. J. Cox, Phys. Rev. B 30, 186 (1984); 30, 197 (1984).

[19] A. Schenck and F. N. Gygax, in Handbook of Magnetic Materials, edited by K.H.J. Buschow (Elsevier, Amsterdam, 1995), Vol. 9.

[20] P. Dalmas de Réotier and A. Yaouanc, J. Phys. Condens. Matter 9, 9113 (1997).

[21] P. Dalmas de Réotier, P. C. M. Gubbens, and A. Yaouanc, J. Phys. Condens. Matter 16, S4687 (2004).

[22] Averaged values are taken for the critical exponents on the basis of MC calculations in Refs. [5,6].

[23] D. Mukamel, Phys. Rev. Lett. 34, 481 (1975).

[24] T. E. Mason, M. F. Collins, and B. D. Gaulin, J. Phys. C 20, L945 (1987); T. E. Mason, B. D. Gaulin, and M. F. Collins, Phys. Rev. B 39, 586 (1989).

[25] H. Kadowaki, S. M. Shapiro, T. Inami, and Y. Ajiro, J. Phys. Soc. Jpn. 57, 2640 (1988); Y. Ajiro, T. Nakashima, Y. Unno, H. Kadowaki, M. Mekata, and N. Achiwa, J. Phys. Soc. Jpn. 57, 2640 (1988).

[26] A. Aharony and G. Ahlers, Phys. Rev. Lett. 44, 782 (1980).

[27] P. C. Hohenberg and B. I. Halperin, Rev. Mod. Phys. 49, 435 (1977). 